\title{
There IS a Question of Physicalism
}

\author{
Orli Dahan*
}

Received: 12 June 2018 / Accepted: 30 November 2018

Abstract: The most common catchphrase of physicalism is: "everything is physical". According to Hempel's Dilemma, however, physicalism is an ill-formed thesis because it can offer no account of the physics to which it refers: current physics will definitely be revised in the future, and we do not yet know the nature of future physics. The dilemma arises due to our difficulty to set the boundaries of the concept 'physical.' In order to confront the dilemma, a physicalist must ensure that physics is not going to broaden itself artificially (or in some trivial way) to become complete - perhaps by adding non-reductive mental entities to elementary physical theory, making it impossible to distinguish physicalism from dualism. I offer a solution to the dilemma which is a version of the 'via negativa' (standardly taken to be a stipulation that the physical not include the mental), albeit one that is specified and worked out in a distinctive way. My suggested formulation of the physicalist hypothesis allows us to establish a refutation condition of physicalism. The refutation condition is general and not only dualistic. Consequently, the physicalist can choose the second horn of the dilemma, and hold that physicalism is indeed refutable (and not a trivial thesis).

Keywords: Completeness of physics; consciousness; dualism; Hempel's Dilemma; physicalism; via negativa.

* Tel-Hai College

Faculty of Humanities and Social Sciences, Tel-Hai College, Upper Galilee, 12208, Israel

$\bowtie$ orlydah@telhai.ac.il

() The Author. Journal compilation (C) The Editorial Board, Organon F.

This article is distributed under the terms of the Creative Commons Attribution-NonCommercial 4.0 International Public License (CC BY-NC 4.0). 


\section{Introduction}

One primary claim of physicalism is that physical theory guides us in answering the question "what does the world contain?" because, according to physicalism, everything is physical. Stated this way, the thesis of physicalism faces a dilemma, referred to in current literature as 'Hempel's Dilemma.' The dilemma, which deals with the meaning of the concept 'physical,' arises because on the one hand contemporary physical theory is incomplete, but on the other hand we know nothing about the character and properties of the future and supposedly complete physical theory. In other words, the question that a physicalist must ask herself, according to the dilemma, is this: which exact physical theory guides the physicalist's ontology? (Hempel 1966; Crane and Mellor 1990).

According to Hempel's Dilemma, there are two ways to interpret the primary claim of physicalism, and both are troublesome:

1. Physicalism is the thesis that the world contains only entities assumed and defined by contemporary physics, and that these entities behave according to the laws of contemporary physical theory.

2. Physicalism is the thesis that the world contains only entities that will be assumed and defined by future physics, and that these entities will behave according to the laws of future-and-complete physical theory.

The first horn of the dilemma leads to a false thesis because contemporary physics is incomplete; at this point, physical theories do not enable us to provide a complete explanation of all that exists in the world. However, the second horn is also problematic because it ostensibly leads to a meaningless, irrefutable, or trivial thesis: we do not really know what we are committed to as physicalists because we do not know enough about future-and-complete physics. The advocate of Hempel's Dilemma is concerned that to become complete, physical science might broaden its scope in different ways, perhaps by adding non-reductive mental entities to elementary physical theory and, consequently, we might not be able to distinguish physicalism from dualism.

Discussions regarding Hempel's Dilemma are common in contemporary literature on physicalism and the mind-body problem [see for example 
(Bokulich 2011); (Crook and Gillett 2001); (Dowell 2006); (Fiorese 2015); (Gillett and Witmer 2001); (Montero 2001); (Ney 2008); (Prelević 2017); (Prelević 2018); (Stoljar 2010); (Wilson 2006); (Worley 2006)]. The responses to the dilemma can be divided as follows: ${ }^{1}$

1. "Currentism": Taking the first horn of the dilemma while dismissing the claim that it makes physicalism a false thesis: some physicalists think that although contemporary physics is incomplete, it is reasonable to assume that its main characteristics will remain more or less the same. For example, Lewis (1983/1999, 33-34) argues that the physical theory that guides ontology is an improved version of contemporary physics that is not exceptionally different from contemporary physics. Bokulich (2011) argues that our knowledge of current physics is sufficient for offering a physicalist ontology of the mind. According to Bokulich, we have solid scientific evidence that future physics will be irrelevant to the mind-body problem because mental processes are part of the well-understood domains of applicability of current physical theories. ${ }^{2}$ Vicente (2011) proposes to construe current physics minimally according to the following assertions: some properties are conserved quantities, those quantities are possessed by bodies, and their distribution and exchange are mediated by forces. According to Vicente, the construal of current physics allows for an adequate definition of the physical with regard to Hempel's Dilemma.

2. "Futurism": Taking the second horn of the dilemma while inserting a constraint on the formulation of physicalism: a popular suggestion is to include in the formulation of physicalism the constraint

1 Prelević $(2017 ; 2018)$ divides the strategies of dealing with Hempel's Dilemma into three: defending currentism, defending futurism or trying to avoid the dilemma by claiming that physicalism is not a thesis. I agree with this general division but add a few more options for rejecting or avoiding the dilemma.

2 Bokulich's approach can be ascribed to a second option: that of choosing the first horn of the dilemma only about the mind-body problem. However, it is worth mentioning that physicalism is a general thesis, which is not limited to the mindbody problem, even if that is the current focus in the literature.

Organon F 26 (4) 2019: 542-571 
"without fundamental mental properties in future-and-complete physics". This solution ensures the possibility of distinguishing physicalism from dualism in the future [see for example (Wilson 2006); (Worley 2006)]. According to Ney (2008), if non-reductive mental entities are included in future-and-complete physical theory, then the dualist will know that she had been right all along, and the physicalist will realize she had been wrong. Therefore, taking the second horn of the dilemma does not make physicalism irrefutable, despite the claim of the dilemma. ${ }^{3}$

3. Avoiding the dilemma by defining the physical as non-mental ('via negativa'): This solution proposes to render the term 'mental' as fundamental and to characterize the physical as 'non-mental,' i.e. defining the term 'physical' in a negative way [for more detail see (Crook and Gillett 2001); (Gillett and Witmer 2001); (Montero 2001); (Montero and Papineau 2005); (Wilson 2006); (Worley 2006)]. This suggestion has much in common with the previous one, and, indeed, the differences between the two solutions are rather subtle. The via negativa solution can be viewed as circumventing the dilemma because it avoids the need to link physicalism to any particular physical theory. Hence, the dilemma becomes irrelevant. Fiorese (2015) argues in favor of the 'via negativa,' holding that either the via negativa is valid, or there is, indeed, no version of physicalism deserving of the name. ${ }^{4}$ Moreover, according to Prelević (2017), although this view was originally introduced as a version of futurism, it can also be incorporated into the hard-core of the physicalist research programme.

4. Avoiding the dilemma by rejecting the claim that physicalism is a thesis: according to Prelević (2017), there are two strategies of avoiding the dilemma by arguing that physicalism is actually not a thesis: the

3 Ney (2008) also argues that physicalism could be better seen as an attitude instead of an ontological thesis and, in this way, to avoid the problems derived from Hempel's Dilemma. Ney's suggested attitude is based upon a commitment to construe one's ontology according to what physics says exists [for a detailed discussion about this view see (Prelević 2017)].

4 For a rather recent criticism of the so-called 'via negativa,' see (Vicente 2011). 
attitudinal approach, according to which physicalism is a stance or an attitude, and the Lakatosian approach, according to which physicalism is best understood as a research programme. If physicalism is not a thesis, it cannot be true, trivial or empty.

5. Rejecting the dilemma: there are several strategies to reject the dilemma while still holding that physicalism is a thesis. For example, Dowell (2006) explains what makes a theory a physical one in terms of the hallmarks of scientific theories, and suggests tying physicalism's ontological commitments to our best methods for justifying our beliefs about the natural world. This solution rules out some entities as falling within the extension of 'the physical' and thus gives 'physicalism' more content than made apparent by discussions of the second horn of Hempel's Dilemma. ${ }^{5}$ Stoljar (2010) argues that the dilemma collapses because it has a third premise (which is almost always overlooked in the literature). The third premise encourages us to choose between the two horns of the dilemma. According to Stoljar, this third premise is mistakenly believed to be a logical truth, while in fact it is not a logical truth, but a substantive falsehood (Stoljar 2010, 105-6). ${ }^{6}$

Each of the proffered solutions is based upon the same basic premise: that empirical physical science will guide us in answering the ontological question of what is in the world. However, I believe that most advocates of Hempel's Dilemma would not resolve the dilemma by reconciling the two horns, as this can be seeing as dodging. Moreover, most advocates of Hempel's Dilemma also would not choose the first horn of the dilemma, no

5 Dowell's view (Dowell 2006) can also be counted as a version of futurism. But I have defined futurism as taking the second horn of the dilemma while inserting a constraint on the formulation of physicalism. Dowell rejects inserting this constraint and, in fact, allows that postulating consciousness at the fundamental level would be in accordance with physicalism. Dowell's view will be discussed further in Section 7 .

6 Stoljar is arguing this from some metaphysical considerations about a twin-physics world (Stoljar 2010, 77). In this sense, his view is different from the view of Dowell (2006), which is more empirically oriented. However, the difference in motivation does not change the result: both views reject the dilemma. 
matter how convincing the idea that current physics will not change significantly, particularly concerning the mind. Quite justifiably, most philosophers will remain unconvinced in the face of the uncertainty of something empirical like physics. At the end of the nineteenth century, it seemed that humankind was close to resolving physical theory only to witness its radical change.

In a way, defining physicalism not as a thesis, but as an attitude or a research programme has many advantages [for a detailed discussion see (Prelević 2017, 2018)]. However, doing so weakens the core idea of physicalism because there is no doubt that physicalism is generally understood as a thesis (or a hypothesis) regarding the actual world. Thus, in this paper, I offer a solution to the dilemma that helps physicalism maintain the status of a thesis.

I believe that a more precise solution, related to the via negativa strategy, exists in the empirical spirit of the solutions mentioned above. To answer the advocate of Hempel's Dilemma, I propose a clarification of the physicalist's primary hypothesis in the context of the mind-body problem and in two other contexts as well (vitalism and emergence). First, in the next section, I briefly discuss physics' pretense to completeness, which further justifies the formulation I am about to suggest, together with its putative implications. In Section 3, I introduce my proposal. In Section 4, I establish a refutation condition of physicalism and argue that it allows us to choose the second horn of the dilemma without making physicalism irrefutable. In Section 5, I show that the refutation condition I offer can disarm the motivation of the advocate of Hempel's Dilemma. In Section 6, I sketch briefly the putative implications of my solution. In Section 7, I emphasize why the way I confront the dilemma is a version of the via negativa, albeit one that is specified and worked out in a distinctive way. In Section 8, I offer concluding remarks.

\section{A note about physics' pretense to completeness}

A remark is needed regarding physics' pretense to completeness. First, because it seems that this is the most successful argument in favor of physicalism (Papineau 2001). Second, because this is the reason for designing 
the physicalist's hypothesis and its refutation condition that I intend to propose.

With regard to the completeness of physics, the point can be phrased in this way: we assume that in order to explain the behavior of billiard balls, tables, hurricanes, and any other parts of the world (objects and phenomena) that do not involve mental aspects - we do not need mental laws (in addition to some complete physical theory). We also assume that the same condition applies in the context of explaining mental states; we do not need additional laws, over and above the laws of complete physical theory, to explain mental phenomena.

Of course, it is another question why (or whether) physics is required (in principle) to explain all there is. What is the source of this universally explanatory motivation? Can we not assume that physics explains some aspects of reality (say - objects like atoms, quarks, tables, and chairs) while accepting that physics (in principle) cannot explain some aspects of other objects (say - objects with a mental aspect, complex objects like organic cells, communities, and such)? This issue is related to 'the causal closure principle of the physical-domain' (CCP), meaning that every physical event has a physical cause [see (Kim 2006); (Stoljar 2017)]. And so, if a behavior of a man (say reaching for a glass of water and drinking) is physical, then it has only physical causes, no matter how strong our intuitions tell us that this behavior also had mental causes (the "will" to drink, or the "feeling" of thirst). ${ }^{7}$

However, the justification for believing in CCP is not obvious. Is it a metaphysical principle (dealing with causality and determination), or is it an empirical hypothesis based on the laws of physics? While according to Papineau (2001) the belief in CCP is historically related to conservation laws, ${ }^{8}$ Montero (2006) rejects that conservation laws ground the CCP, and

7 A non-eliminativist physicalist will insist that the behavior did indeed have mental causes because the mental is physical. Of course, there are no causes in addition to the physical causes.

8 In physics, a conservation law states that a particular measurable property of an isolated physical system does not change as the system evolves. Laws that have been confirmed so far (i.e. never been violated) include conservation of mass-energy, conservation of linear momentum, conservation of angular momentum and conservation 
Vicente (2006) tries to explain how the CCP could be made to follow from conservation laws using additional premises. On the other hand, Bishop (2006) argues that there is a hidden premise that must be added to the CCP for the causal argument to be sound. The hidden premise is as follows: 'the only efficacious states and causes are physical ones.' But since it is indistinguishable from the conclusion of the $\mathrm{CCP}$ - the argument begs the question regarding physicalism.

Nevertheless, the pretense of physics to completeness can be phrased differently. For example, if the "Theory of Everything" (the physical theory that would account for everything that there is) has been given to us (in some miraculous way), then the claim of the physicalist is: there is no extra fact in the world that is not derived from that theory. ${ }^{9}$ Kripke phrases physicalism's claim in a similar way, though much more figuratively. According to Kripke, if physicalism is true, then when God created the world, God had only to fix the elementary particles and set the relationship between them, and everything else occurred automatically (Kripke 1980, 153-54).

In this context, we can see the tight connection between the ideal physical theory, physics' pretense to completeness, and the metaphysical thesis of physicalism. The physicalist thinks of mental states, in particular, and all other states, in general, as included in the complete physical theory about the world. Certainly, the thesis of physicalism regarding the mindbody problem is not yet confirmed, because it is only a hypothesis: ${ }^{10}$ at the moment we do not possess a psycho-physical theory. However, even if physicalism is a hypothesis, it still might be a highly-confirmed hypothesis. Because for physicalists who accept the causal closure argument as sound-it

of electric charge. Additional conservation laws have been confirmed empirically; however, the subject goes beyond the scope of this paper.

9 See for example (Redhead 1996, 63-66) on the "theory of everything" as the Holy Grail of modern theoretical physics. The unification programme of fundamental physics will be discussed further in Section 4 .

10 One can ask whether this hypothesis is metaphysical or empirical. Typically, physicalism is a metaphysical view of the nature of reality (or at least this is the way it is discussed within the mind-body literature). However, others think of physicalism more like an empirical hypothesis regarding the actual world [see (Spurrett 2017)]. 
seems to provide good confirmation of physicalism even in the absence of a psycho-physical theory.

I want to emphasize that in this paper I have no intention of arguing in favor of physicalism. The aim of this paper is only to show that there is, in fact, a question of physicalism - thus the thesis of physicalism is not trivial. I argue that in the future, the thesis of physicalism might be discovered to be false. For this reason, I am not going to justify physics' pretense to completeness or the CCP; for these subjects go beyond the scope of this paper and are indeed in controversy. However, in the context of the completeness of physics, it is worth noting that even in the many diverse branches of physics itself, were we to assume a successful reduction, the reduction would not necessarily be complete. One such branch is thermodynamics: we still cannot derive all thermodynamics laws and phenomena from classical statistical mechanics [for further details see (Hemmo and Shenker 2012)].

And so, there are many arguments and discussions in the literature of the mind-body problem against the thesis of physicalism. Moreover, there are many other issues related to the field of philosophy of science in general that question whether physics can reach completion or can, in general, explain all there is (opponents include strong emergentists). However, the strength of Hempel's Dilemma is its second horn, that physicalism is an empty doctrine and not refutable under any future conditions and circumstances. This claim, I will show, is wrong.

\section{The hypothesis of physicalism elucidated in the context of Hempel's Dilemma}

I will introduce my formulation of the hypothesis in the context of the mind-body problem while noting that physicalism is a general thesis and the hypothesis can be phrased in other contexts as well (for example, in the context of the relationship between physics and biology or physics and aesthetics). Of course, the consequences of Hempel's Dilemma reach beyond the philosophy of the mind; if the dilemma is sound, it potentially invalidates physicalism as a thesis that is false or has no content at all. A crucial note is that this formulation by itself is not an answer to the dilemma, but 
only the first step in the way to construct a refutation condition of physicalism (in the next section).

The hypothesis of physicalism from the mind-body point of view, based on the supervenience thesis, ${ }^{11}$ emerges out of two premises:

1. Premise I: regarding the world - the macroscopic objects in the actual world can be divided into two sets: set a includes things without mental properties (for example tables, chairs, and even complex systems and phenomena, such as hurricanes or cells, as long as they do not possess any mental states) and set B includes things with mental properties (for example humans). These two sets exhaust the macroscopic objects that exist in the actual world.

2. Premise II: regarding the future-and-complete physical theory - the laws of future-and-complete physics will be identical for both objects of set $\mathrm{A}$ and of set $\mathrm{B}$.

3. Therefore, physicalism's primary hypothesis is as follows: the physical laws of a will (in principle) be sufficient in order to serve as a foundation for building psychological theories. Meaning: the physical laws that apply to objects in set a are sufficient to describe and explain the behavior and properties of objects in set B. ${ }^{12,13}$

11 Supervenience is a relation that is used to describe cases where the upper-level properties of a system are determined by its lower level properties. Most philosophers think of physicalism as a metaphysical thesis, and so it is usually understood to mean that everything logically (or metaphysically) supervenes upon the physical. Others believe the supervenience thesis with regard to physicalism is contingent because physicalism is simply an empirical hypothesis about the actual world. However, this observation is not crucial regarding my argument in this paper.

12 Also, they will be sufficient for building the foundation of any other scientific theory, for example, thermodynamics, biology, geology, etc.

13 This formulation is, in fact, compatible with epiphenomenalism. If mental properties are epiphenomenal, then it will be possible to describe and explain the behavior of objects in set B using physical laws, even though there will be some properties that are not physical. But there are a few reasons to think that epiphenomenalism is not an option for the actual world. For example, Papineau (2016) argues that epiphenomenalism is not an attractive position, for it presents a very 
This formulation can be seen as a 'via negativa' way to characterize the physical domain; however, I emphasize that this is not the only way of dividing the macroscopic world in an exhaustive manner. One can use other sets of objects to divide the world in an exhaustive manner. Given the context of the mind-body problem and physicalism, I present the first premise this way. From of a biological point of view, I would divide the objects in the world differently.

In fact, I propose to handle the advocate of Hempel's Dilemma by relating the physical to the macroscopic objects and properties that physics is bound to account for, and not by rendering the term 'mental' as fundamental and by characterizing the physical as 'non-mental.' The recent tendency to focus on Hempel's Dilemma as an issue for physicalism about the mind neglects the importance of vitalism and emergence for the history of physicalism as well as the thinking about how to distinguish the physical from the non-physical. At times, this focus makes the topic of physicalism seem needlessly parochial or narrow. For this reason, I suggest two additional ways of formulating the physicalist's hypothesis: a vitalist and a strong emergence formulation.

The hypothesis of physicalism from a biological point of view, based on the supervenience thesis, emerges out of two premises:

odd kind of causal structure: "nature displays no other examples of such one-way causal intercourse between realms." Moreover, there is an epistemological problem with epiphenomenalism: we cannot explain our knowledge about mental states if mental states are just epiphenomenal and have no effect on the world (BraddonMitchell and Jackson 1996, 6-7). And, if mentality is not a basic structure of the world, but an evolutionary product (or by-product), it seems hard to explain from an evolutionary point of view the existence of properties that have no effect on us (Braddon-Mitchell and Jackson 1996, 6-7). Given these considerations, it seems reasonable to exclude the epiphenomenalism possibility about the actual world, or even just to prefer physicalism over epiphenomenalism. I am aware of the fact the one cannot be comfortable with an account that allows for the possibility of there being epiphenomenal non-physical properties. However, one should acknowledge that the possibility that something non-physical obeys physical laws is remote or directly nonsensical. For more detail about epiphenomenalism see (Robinson 2015). 
1. Premise I': regarding the world - the macroscopic objects in the actual world can be divided into two sets: set A' includes things without organic properties (for example tables, chairs, and even complex systems and phenomena, such as hurricanes, as long as they do not possess any organic properties) and set B' includes things with organic properties (for example humans, amoebas, and bacteria). These two sets exhaust the macroscopic objects that exist in the actual world.

2. Premise II': regarding the future-and-complete physical theory - the laws of future-and-complete physics will be identical for both objects of set $\mathrm{A}^{\prime}$ and of set B'.

3. Therefore, physicalism's primary hypothesis is as follows: the physical laws of A' will (in principle) be sufficient in order to serve as a foundation for building biological theories. Meaning: the physical laws that apply to objects in set A' are sufficient to describe and explain the behavior and properties of the organic objects in set B'. ${ }^{14}$

The hypothesis of physicalism from an emergentist point of view, ${ }^{15}$ based on the supervenience thesis, emerges out of two premises:

14 This version of physicalism can be seen as similar to a venerable proposal by Meehl and Sellars (1956) that urged distinguishing a very broad and a more restrictive version of the 'physical' (the ' 1 ' and '2' are sub-scripts in the original): physi$\mathrm{cal}_{1}$ - terms employed in a coherent and adequate descriptive, explanatory account of the spatiotemporal order; physical ${ }_{2}$ - terms used in the formulation of principles which suffice in principle for the explanation and prediction of inorganic processes. Meehl and Sellars (1956) were also concerned with emergence and wanted to reject an argument that the doctrine is trivially false, arguing instead that it is coherent (and empirically false).

15 According to Chalmers (2006), the term 'emergence' is used to express at least two different concepts: weak emergence and strong emergence. Weak emergent properties are (in principle) deducible from the low-level properties (perhaps in conjunction with knowledge of initial conditions) while strongly emergent phenomena are systematically determined by low-level facts without being deducible from those facts. Thus, strong emergence has much more radical consequences than weak emergence: "Strong emergence, if it exists, can be used to reject the physicalist picture of the world as fundamentally incomplete. By contrast, weak emergence can be used to support the physicalist picture of the world by showing how all sorts of phenomena 
1. Premise I": regarding the world - the macroscopic objects in the actual world can be divided into two sets: set A" includes things without emergent properties (for example tables, chairs, and all objects that do not introduce emergent properties) ${ }^{16}$ and set B" includes things with emergent properties (for example hurricanes, humans, traffic jams, and communities). These two sets exhaust the macroscopic objects that exist in the actual world.

2. Premise II': regarding the future-and-complete physical theory - the laws of future-and-complete physics will be identical for both objects of set A" and of set B".

3. Therefore, physicalism's primary hypothesis is as follows: the physical laws of A" will (in principle) be sufficient in order to serve as a foundation for building high level theories about emergent objects and phenomena in the world. Meaning: the physical laws that apply to objects in set A" are sufficient to describe and explain the behavior and properties of the emergent objects and properties in set B".

\section{Establishing a refutation condition of physicalism and choosing the second horn of the dilemma}

According to the aforementioned formulation of the physicalist's claim, I will now explain why a physicalist can choose the second horn of Hempel's

that might seem novel and irreducible at first sight can nevertheless be grounded in underlying simple laws."

16 It is worth noting that according to some views, tables and chairs can also be viewed as objects that introduce emergent properties [see for example (Teller 1992)]: the macroscopic properties of a table is much different from its microscopic properties. If we take the "naked" emergentist intuition to be that an emergent property of a whole somehow "transcends" the property of the parts - nearly all macroscopic scale objects would be "emergent". If this is the view in our context, then the hypothesis of the physicalist can be formulated slightly different, and divide the world into microscopic objects and macroscopic objects. However, I chose to construct the hypothesis as follows because most strong emergentist's views would not consider tables as emergent in an interesting way [see also (Chalmers 2006).

Organon F 26 (4) 2019: 542-571 
Dilemma while rejecting the claim that it makes physicalism irrefutable. When looking at premise II, it becomes clear that if in the future premise II would be refuted, then physicalism would be refuted as well. The physicalist claims that the laws of physics govern the bodies and behavior of humans, and also govern the bodies and behavior of objects such as tables, chairs, billiard balls, and even more complex phenomena such as hurricanes or cells. This is the reason why the parts of the world in which mental states are present (objects of set B) are approximately governed by the laws of contemporary physics. If this hypothesis is to be refuted in the future, for instance, due to a division of physical laws between set $\mathrm{A}$ and set $\mathrm{B}$, or between set A' and set B', or between set A" and set B" - - then physicalism is refuted. Now, because the dilemma asserts the falsehood or triviality of physicalism, in formulating the thesis as in the previous section, I maintain that the thesis of physicalism is not trivial at all. The thesis has meaning. It also seems that physicalism cannot be dismissed offhandedly as false (as according to the first horn of the dilemma) because formulated this way, the physicalist's hypothesis provides stipulations for its confirmation as well as its refutation.

The refutation condition of physicalism can be formulated as follows:

If the laws of complete physics are divided between two sets of objects that exhaust the macroscopic objects that exist in the actual worldthen physicalism is refuted. ${ }^{17}$

17 The two sets must be divided with respect to the macroscopic non-physical properties of the objects in the actual world (as in the three examples discussed in Section 3 ) due to physics' pretense to completeness (discussed in Section 2). Otherwise, we could divide the objects in the world between charged objects and uncharged objects: charged objects require the physics of electromagnetism, while uncharged objects require only Newtonian mechanics. But we would not consider this a refutation of physicalism. Physicalism is the claim that fundamental physics, in principle, can explain all there is in the actual world - even what seems to us in the macroscopic world as not physical (such as the mental, the biological, the moral, and the aesthetic). The question about the unification of physics itself is a different question, separate from the question raised by Hempel's Dilemma (although interestingly related - see a brief discussion at the end of this section). For example, if physics discovers that there are two physically different categories of physical entities - such as 
But in any other case that the laws of complete physics are not divided between any two sets of objects that exhaust the macroscopic objects that exist in the actual world - however strange and counterintuitive it may appear-we must admit that physics has succeeded to explain all there is in the world, and became complete in a non-trivial way. ${ }^{18}$

Let us consider the case that future-and-complete physics will assume special particles, say mental ones. According to the formulation I have proposed, this category of particles poses no dilemma as long as the situation is consistent with the second premise. That is, if these "extra" fundamental entities or forces are required and assist not only in explaining psychological phenomena (objects in set B), but also in explaining billiard balls (objects in set $\mathrm{A}$ ), then this is consistent with the supervenience of everything there is in the world upon physics. In this case, the existence of mental entities does not necessarily challenge physicalism.

Moreover, we can imagine a case in which supervenience is violated. For example, in the future, unique explanations, laws, or entities of physical theory may be needed to explain mental phenomena (objects in set B). More precisely, the existence of explanations, laws, or entities will be necessary in addition to the laws and entities that are sufficient to explain objects in set A. In this case, the physicalist's hypothesis would be refuted, and physicalism will be proven false.

The same is true for the other sets as well: we can imagine a case in which supervenience is violated, not only from a dualistic point of view. For example, in the future, unique explanations, laws, or entities of physical

matter and anti-matter - we would not consider this a refutation of physicalism. The refutation condition helps us argue that physicalism is not a trivial thesis, and that the subject matter in the refutation condition is macroscopic objects and not fundamental physical entities.

18 One can ask the following question: does this mean that, for all we now know, physicalism is false? After all, we seem to have a division between the physics of the micro and the physics of the macro. The answer is that this division is precisely one of the reasons (among others) demonstrating that contemporary physics is incomplete. Physics is uncomfortable with this division in its laws and tries to find a unified theory in order to resolve this division. And so, the answer to the question is negative: we know nothing about future and complete physics, and we cannot jump to conclusions regarding the falsity of physicalism based only on current physics. 
theory may be needed to explain organic phenomena (objects in set B'). More precisely, the existence of explanations, laws, or entities will be necessary in addition to the laws and entities that are sufficient to explain objects in set A'. In this case, the physicalist's hypothesis would be refuted, and physicalism will be proven false. Supervenience can also be violated from an emergentist point of view. For example, in the future, unique explanations, laws, or entities of physical theory may be needed to explain emergent phenomena (objects in set B"). More precisely, the existence of explanations, laws, or entities will be necessary in addition to the laws and entities that are sufficient to explain objects in set A". In this case, the physicalist's hypothesis would be refuted, and physicalism will be proven false.

My suggestion tackles the case in which a future unified physical theory is achieved in a trivial way. For instance, future and complete physics could simply be a conjunction of two theories: a physical theory and a psychological theory / a physical theory and a biological theory / a physical theory and a theory regarding complex systems. If a physicalist, a dualist, a vitalist, or an emergentist would examine the alleged unified physical theory carefully, they would discover that the laws of "the final and complete physical theory" are divided between two sets (depending upon which point of view the division of sets was made) of objects and phenomena in the world. For example, there are a few allegedly fundamental laws of physics that explain the organic or mental parts of the world, but not the non-organic or non-mental parts of the world. Likewise, there are a few allegedly fundamental laws of physics that explain the emergent aspects of reality but not the non-emergent aspects of reality. If this is the case, the physicalist, dualist, vitalist or emergentist would know that the alleged unified physical theory is unified in a trivial way and physics is not truly complete. Hence, the physicalist hypothesis is refuted.

Those who think this proposal for examining future unified physical theory is farfetched may consider that it is common in the literature to question the alleged successful unifications in theoretical physics achieved to date. In short, four basic physical forces exist in fundamental physics: electromagnetism, gravity, the strong nuclear force, and the weak nuclear force. Theoretical physics is attempting to produce a theory unifying these forces. Its aim is to demonstrate that there is only one fundamental force in the 
universe. The first step in this unification programme has already been achieved: electromagnetism has been unified with the weak nuclear force in the electroweak theory. Next, the electroweak force is to be unified with the strong nuclear force by a grand unified theory (GUT). Finally, the GUT will be unified with gravity in a Theory of Everything [TOE; (Maudlin 1996)].

But philosophers and physicists dare to question this particular unification programme. For example, Rescher (1999) claimed that combining the four fundamental forces is insufficient because the 'Theory of Everything' must be holistic, and not simply an aggregation of forces. According to Maudlin (1996), the image of this unification programme has become so pervasive as to rank almost as a dogma. Why assume that these four forces are to be unified other than for purely aesthetic reasons?

Furthermore, Maudlin (1996) demonstrated that the unification of the weak nuclear force and the electromagnetic force was forced on those who were primarily engaged in seeking an adequate theory of the weak force. In fact, some theorists (for example Richard Feynman) deny that the electroweak theory displays any real unification of electromagnetism and the weak force: "it is not that at some point we had theories of the electromagnetic, weak, strong, and gravitational forces separately, and now we have managed to unify the first two. Rather, at some point, we recognized the existence of all four forces, and found that unification was needed to account for the weak force" (Maudlin 1996).

These examples show that even in the realm of fundamental physics we are asking questions regarding "real" and "holistic" unification, as opposed to just "aggregations" or "combinations". My formulation of the physicalist's hypothesis and its refutation condition is designed to answer similar considerations. One can see the resemblance between the point of view introduced in Hempel's Dilemma and the skeptical point of view about unifications in physics itself.

\section{Understand the dictation of the refutation condition}

It seems that the advocate of Hempel's Dilemma is familiar with physics' pretense to completeness, and hence is troubled about the broadening of physics for the sake of its completion. Of course, most advocates of Hempel's 
Dilemma are concerned about the addition of mental aspects to physics in order to explain the mental parts of the world. Advocates from other perspectives can formulate similar dilemmas. For example, a hypothetical vitalist would state that physicalism is an ill-formed thesis: it is obvious that we do not yet have a complete physical explanation for every biological phenomenon (meaning that biology is still not fully reducible to physics), so it could be that for the sake of completeness, future physics will be expanded to include some non-reductive biological entities or forces. This makes physicalism, in the eye of this hypothetical vitalist, a false or unrefuted thesis. ${ }^{19}$

A more important point, perhaps, is that the physicalist can do nothing about it. The physicalist cannot set rules regarding the characteristics of the final entities and laws of physics. For this was the lesson physicalists learned from their predecessors: calling the thesis materialism was a mistake, resulting from the historical belief that physics deals only with matter. Today we know that the stuff of physics includes energies, forces, fields, and entities whose composition remains a mystery (particles, strings, membranes, or perhaps something else). A physicalist who understands the dependence of his thesis on the empirical content of physical science cannot characterize the nature of ultimate physical theory. To do so would be to ignore the end of materialism.

However, the physicalist can ensure that physicalism does not become a trivial thesis. One method for achieving this objective is to prohibit future splitting in the laws of physics between any two sets of objects that exhaust the macroscopic objects that exist in the actual world. For this reason, I constructed the physicalist's hypothesis as presented in Section 3. If a division would occur between the laws that govern objects and properties from set $\mathrm{B}$, and the laws that govern objects and properties from set A, then we could assume that the additional laws are not part of physics, but were added from outside of physical theory in order to explain the

19 This is just a hypothetical example - I do not imply a necessary connection between reductionism and Hempel's Dilemma. One can argue for physicalism without having reductive explanations in hand, and it seems that if the argument of the dilemma is sound, then even having such reductions in hand would not allow one to claim that everything is physical. Hempel's Dilemma seems to be quite independent of any worries about the (lack of) success of reduction to physics. 
mental phenomena. If a division would occur between the laws that govern objects and properties from set B', and the laws that govern objects and properties from set A', then we could assume that the additional laws are not part of physics, but were added from outside of physical theory in order to explain the biological. And if a division would occur between the laws that govern objects and properties from set B", and the laws that govern objects and properties from set A", then we could assume that the additional laws are not part of physics, but were added from outside of physical theory in order to explain the emergent phenomena of the world. In each of these cases, the physicalist's hypothesis would be refuted. Also, the dualist / vitalist / emergentist would be able to say that she was right all along because physics alone cannot explain the mental / organic / emergent aspect of the world.

For any other case, such as that of mental entities (or laws) in physics, which are needed in order to address or explain objects and properties from set A and set B, the physicalist should not care at all. The same is true in the case of a "vital force" in physics needed to address or explain objects and properties from set A' and set B'. I argue that the physicalist should not care at all even if a new law is needed to address or explain objects and properties from set A" and set B". On the contrary, in the case of mental entities / vital force / emergence law, it could then be said that physics succeeded in uncovering the true structure of the world. For this is exactly what the physicalist assumes: it is the task of physics to discover the nature of the world and how that nature explains every manifestation in the world. This notion stems from understanding physicalism as a metaphysical hypothesis that is related tightly to the actual world. The physicalist cannot veto the character and nature of the content of physics. This is the crucial point of the dilemma.

The constraint regarding the un-splitting of laws that govern the objects and properties in the world is not external to the thesis. More importantly: this constraint is general and does not focus only on the mental aspect. These two aspects of the un-splitting constraint are opposed to the constraint "without mental entities in future and complete physics." My constraint is generated from within the thesis. The physicalist should not be concerned with physics' articulation of the structure of the world. She 
should care whether physics succeeds in uncovering that structure without being artificially completed by laws and entities.

\section{What should we learn from Hempel's Dilemma?}

I suppose most philosophers will still have difficulty accepting that physicalism can be true even if the future-and-complete physical theory includes fundamental mental entities (whatever they may be), and even if these entities meet the demands I introduced earlier (i.e. that those entities would be required for explaining phenomena and objects in both sets, A and B). Even the vast majority of physicalists may have difficulty accepting this idea. I realize how controversial this may sound: it can be argued that the notion of physicalism that I provide does not correspond to the use of the word 'physicalism' in the philosophical community (or at least in the way it is discussed within the mind-body literature). For typically, the physicalist rejects fundamental mentality. ${ }^{20}$ But the typical physicalist also rejects fundamental biology and more fundamental stuff that are not physical. This prompts the question: what does count as 'physical'? And obviously, we have returned to the dilemma.

In any case, these proffered suggestions may be the real solutions to the dilemma, stemming from the elucidated hypothesis of physicalism and its refutation condition. However controversial, as physicalists we must note that the possibility that future and complete physics will contain "mental" entities, or "emergent" laws, or "vital" forces (whatever the meaning of these expressions may be in the future) is negligible. Nevertheless, the point is that being physicalists, we cannot a-priori rule out these possibilities completely. As argued by Spurrett (2017): "The fact of revision and revolution in the history of science, and the undoubted provisionality and incompleteness of science as we have it, do indeed tell against simply letting current science determine what the physical (or material) is for philosophical purposes." In my opinion, the crucial point is the physicalist's commitment

20 Prelević (2018) discussed the concern that postulating consciousness at the fundamental level is not in accordance with standard classifications in the history of philosophy (for further discussion see Section 7). 
to physics, derived from the understanding of the motivation for being a physicalist and the point-of-view introduced in the dilemma.

We may want to think about the following question: is the purpose of the project to provide a notion of physicalism that makes sense of the debate and clarifies what philosophers have in mind when they are saying 'physicalism,' or is it more like a normative project, providing this notion of physicalism as the one that we should use? I see myself as a physicalist from naturalisticempirical arguments. But the dilemma regarding the notion of the word 'physical' and its boundaries has always troubled me. I am obviously not offering here a solution to the question "what physics is meant?" but that is exactly because of the empirical nature of science and physics.

Either way, from a physicalist point of view, how can we set boundaries to the notion physical and still hold that "everything is physical"? After all, if everything is physical, then the line between physics and chemistry is only an arbitrary convention that can be moved according to our needs (or, the line was set by us this way because of our physical brains that make us see the world divided in such and such manner), as is the line between chemistry and biology, and so on. From this point of view, the project of capturing the notion 'physical' is paradoxical in its essence and is bound to fail. Trying to capture an empirical notion such as 'physical' contradicts the essence of the empirical sciences.

\section{A note regarding the other solutions to Hempel's Dilemma}

Dowell (2006) and Vicente (2011) criticize the use of via negativa, maintaining that it is unnecessary to include the mental in the definition of the physical out of skepticism regarding a priori truths in general. I agree with this view. My use of the via negativa in the physicalist's hypothesis is only partial. I divided the world into two sets of objects, set a containing objects that do not possess mental properties, and set B containing objects that do possess mental properties. While this initial step is, in and of itself, use of via negativa, one should continue and elucidate the physicalist's hypothesis to answer the dilemma. In other words: one should use the refutation condition of physicalism. As noted above, it is obvious that the dilemma can be articulated from other points of view. If the vitalist would ask "what is 
physical?" and the answer would be via negativa, "not biological", this surely is not a sufficient solution. If the physical is not mental, and not biological, and not aesthetic, and not moral, and so on - then what is the physical? Apparently, we are left with the answer: "the physical is physical"... and our dilemma remains unresolved. The crucial point is this: my suggested construction of the physicalist's hypothesis is only a pragmatic step in the purpose of formulating a refutation condition of physicalism.

Furthermore, setting aside what to classify as the 'via negative,' it is worth noting that before that term became current, some had urged pragmatic flexibility about what to exclude from the physical. Spurrett and Papineau (1999) took an overtly pragmatic line, saying "[which] completeness thesis you ought to be interested in thus depends on the purpose to which you want to put the causal argument." (A 'completeness thesis' refers to a causal closure principle.) In later work, Papineau (2001) also urged flexibility about how to apply the 'ideal physics as long as it does not include X' template, saying: "The same point applies if we want to apply the causal argument to chemical, or biological, or economic states. As long as we can be confident that all non-chemical effects are fully caused by nonchemical (non-biological/non-economic...) states, then we can conclude that all chemical (biological/economic...) states must be identical with something non-chemical (non-biological/non-economic...)." ${ }^{21}$

However, the line suggested in (Spurrett and Papineau 1999) and in Papineau's (2001) more pragmatic remarks, is not how most philosophers understand the 'via negative.' Hence, my offer, rather than being radically new, picks up a neglected line of thought and shows how it has traction in the contemporary literature on Hempel's Dilemma.

On a related point, it is worth noting a slightly obscure form of vitalism associated with Walter Elsasser (1958; 1962), according to which the laws of 'physics' (the science of the inanimate) were special cases of the laws of the animate. However, Elsasser was not a pan-psychist, although he may in some sense have been a pan-biologist or pan-vitalist. His relevance here is that his view suggests a different set of solutions for the template developed in this paper about laws and objects. Elsasser thought that there was

${ }^{21}$ I thank an anonymous referee for this line of thought. 
a complete science of all objects and that it had 'biotonic' laws. But it was not 'physics,' and he rejected what he called 'materialism. ${ }^{22}$ The trivial physicalist betting on future/ideal physics would, it seems, attach the label 'physical' onto the 'biotonic' laws. The non-mentalist proponent of the 'via negativa' would, perhaps, do so as well. However, it is not clear that Papineau (2001) would want to follow suit, and I believe it is clear he would say that in an Elsasser world, physics is not complete. However, in my opinion, and according to the formulation and the refutation condition I have offered for physicalism - it is possible that an Elsasser world is a world compatible with the physicalist hypothesis.

As for both Ney's (2008) solution and the suggestion to include some constraints in the future and complete physical theory, I have shown that there is a possibility that physicalism actually permits such "mental stuff" (or "vital stuff," or any other stuff) in fundamental physics, as long as such inclusion is consistent with the physicalist's hypothesis. Hence, it seems that there could be a hypothetical case in which both physicalist and dualist would be saying that they had been right all along. They would both be right, in a way. The physicalist would have been right when claiming that physics will uncover the true nature of the world and that all 'God' had to do was to create the entities and laws, and everything else would come into existence automatically. The dualist (or panpsychist) would also have been right since mentality is in the fundamental stuff of the world. ${ }^{23}$

Dowell (2006) regarded the physical not as metaphysical but as methodologically empirical. Dowell suggested tying physicalism's ontological commitments to our best methods for justifying our beliefs about the natural world: TOE should be a theory with the hallmarks of scientific theories.

22 For more about Elsasser's view see (Bronowski 1970) and (Gatherer 2008).

23 I admit that this suggestion raises concern: maybe the mental is fundamental, for example in the form of panpsychism. Then it would not be true that we need a separate set of laws to govern the mental and the physical and, in my view, physicalism would not be refuted. I suggest that this is a bullet that we might have to bite. It is not for us to decide, a priori, what physics will end up looking like, or what the world will turn out to be. It is certainly true that we cannot say, a priori, what physics will contain. And one does not want this to turn into a mere verbal dispute about the use of the term "physical."

Organon F 26 (4) 2019: 542-571 
Dowell (2006) also opted for a future physical theory that postulates consciousness at a fundamental level in accordance with physicalism, without making this view trivial or empty. Prelević (2018) discussed the concern that postulating consciousness at the fundamental level is not in accordance with standard classifications in the history of philosophy and, in fact, may allow classifying philosophers like Descartes, Leibniz and perhaps Chalmers as physicalists, which seems implausible. However, I believe the refutation condition I offer may help clarify this concern. Although I agree with Dowell, I believe that, in a way, my view expands Dowell's view by providing a refutation condition of physicalism that does not require a strict notion of the physical that opposes the empirical essence of physics. Furthermore, using my refutation condition, not all future TOEs postulating consciousness at the fundamental level will be in accordance with physicalism-even if they have the hallmarks of scientific theories. In this respect, my view is distinguished from the view of Dowell (2006).

Of course, if at the end of physics we find ourselves considering the "Theory of Everything" from the dualist point of view, and use my refutation condition to ensure that the unification is not trivial, there will be an inherent risk. Emergentists about complex entities will then classify as physicalists, for if hurricanes and cells make it into set $\mathrm{A}$, then no matter what view you have about them, your view will ipso facto count as physicalist. The same risk is inherent for some vitalists, for if viruses and bacteria become part of set A, then no matter what view you have about them, your view will ipso facto count as physicalism.

My answer to this quandary is this: it is possible that as soon as we have the TOE, we will need to start using my refutation condition from several points of view to ensure that the unification was not achieved in some trivial way. We will have to examine carefully the laws of the final physical theory by dividing the world into various sets (such as set A' and B', and A" and B" ${ }^{24}$ to ensure that the laws of physics are not divided. We might find, for example, that physics has succeeded in explaining mentality but failed to explain other complex entities. In that imaginary scenario, although (perhaps) there will be no fundamental mentality in physics, we will still be

24 There can be many other possible sets in future science of which we are unable to conceive at this point in time. 
able to argue (using my refutation condition) that physicalism is false from an emergentist's point of view.

A good example of a new point of view is Integrated Information Theory (IIT), one of today's most influential theories regarding consciousness. Applying my refutation condition of physicalism to the case of IIT can be helpful in comparing the approach in my paper to other prominent solutions to the dilemma. ${ }^{25}$ According to IIT, consciousness is integrated information, meaning that the quantity of consciousness corresponds to the amount of integrated information generated by a complex of elements, and the quality of experience is specified by the set of informational relationships generated within that complex (Tononi 2008). This theory has ontological consequences: according to IIT, integrated information exists as a fundamental quantity - as fundamental as mass, charge, or energy. Since consciousness is the same thing as integrated information, IIT argues that consciousness itself is a fundamental property (Tononi 2008). So, concerning Hempel's Dilemma, is IIT compatible with physicalism? Alternatively, in the event that we acquire empirical justification for IIT in the future, we will conclude that physicalism is a false thesis?

It seems that according to the via negativa we can answer immediately, even without waiting for empirical justification: since according to the via negativa we define the physical as non-mental, if we find out that indeed the physical can also be mental in some cases (i.e., integrated information), then physicalism is false. But when applying my refutation condition to this particular case, the answer is not so straightforward. For in the case of IIT, we can imagine a situation in which supervenience is violated. For example, in the future, unique explanations, laws, or entities of physical theory may be needed to explain computational properties or computational phenomena. More precisely, explanations, laws, or entities will be necessary in addition to the laws and entities that are sufficient to explain objects without computational properties. Of course, the opposite case is also possible: let us consider the case that future-and-complete physics will assume laws regarding these so-called fundamental integrated information properties

25 It would also be interesting to apply my refutation condition of physicalism to some dualistic interpretation of quantum mechanics [for example see (Barrett 2006)]. However, this is beyond the scope of the current paper.

Organon F 26 (4) 2019: 542-571 
(whatever they will be). According to the formulation I have proposed, this category of fundamental laws and properties poses no dilemma, if these „extra" fundamental entities / forces / laws / properties are required and assist not just in explaining brains and computers, but also in explaining tables, chairs, and rocks, then this is consistent with the supervenience of everything there is in the world upon physics. In this case, the existence of integrated information properties in the fundamental level of the world does not necessarily challenge physicalism.

Developing this example is beyond the scope of the current paper. Nevertheless, this example of IIT emphasized the point that the other solutions to the dilemma (such as the via negativa and futurism) are quick in judging a-priori what we should find in the fundamental level and what we should not while ignoring the empirical characteristics of physics itself. I believe that my solution leaves both options open while not avoiding the problem or ignoring the possibility that future physics will be entirely different than we can imagine.

\section{Conclusion}

This paper joins the ongoing argument over how best to respond to what has become known as 'Hempel's Dilemma,' the choice facing physicalists over whether, when they say "everything is physical" they mean current physics or ideal/future physics. I defend a version of the respondents saying that the 'future/ideal' physics option can be selected without the consequence of making physicalism trivial. The triviality consequence is avoided because of the inclusion of a 'refutation condition.' This refutation condition is a version of the 'via negativa' (standardly taken to be a stipulation that the physical not include the mental), albeit one that is specified and worked out in a distinctive way. Thus, the solution offered here can be described as a new member of an established family of strategies: picking up a neglected line of thought and showing how it has traction in the contemporary literature on Hempel's Dilemma.

Hempel's Dilemma arises due to the most common catchphrase of physicalism "everything is physical," and our difficulty stabilizing the extension of the concept physical. It seems that the advocate of Hempel's Dilemma is 
concerned that physics will broaden itself artificially to become complete. Thus, the dilemma is not about distrust of physicalism. The dilemma presents a concern about not trusting physics. Moreover, the physicalist is powerless to prevent physics from artificially broadening for the sake of becoming complete.

I have suggested that in order to confront the dilemma, we must first clarify the physicalist's hypothesis, thereby dismissing ambiguous definitions. What the physicalist can do is to ensure that physicalism is not a trivial thesis by strengthening the physicalist's hypothesis to address the concern raised by Hempel's Dilemma. The hypothesis suggested is that in future and complete physics there will be no splitting in the laws between the set of objects possessing mental properties and the set of objects not possessing mental properties (or any other two sets according to which we choose to divide the objects in the world). If a splitting took place, both dualist (or any other opponent to physicalism) and physicalist would know that physics has failed in its attempt to become complete and that physicalism is refuted.

My approach differs from others not only because of its suggested refutation condition for physicalism but also through its direct attention to vitalism and emergence. The recent tendency to focus on Hempel's Dilemma as an issue for physicalism about the mind neglects the importance of vitalism and emergence for the history of physicalism and for thinking about how to distinguish the physical from the non-physical.

By providing a more accurate answer to Hempel's Dilemma - a general refutation condition of physicalism - I bring to light the understanding about that to which the physicalist is committed. Even if this commitment is but a fraction more than what appears in the dilemma, it would be sufficient to show that physicalism is not a trivial thesis. In other words: there IS a question of physicalism.

\section{Acknowledgements}

I am very much indebted to Meir Hemmo for many stimulating discussions of these matters. I am also grateful to Yael Raizman-Kedar, who provided extensive written feedback on an earlier draft, and to David Buzaglo, Aya Evron, and the attendees of the 2016 "Cognition Research Seminar" at Haifa University for a helpful discussion. Many thanks to the anonymous reviewers for Organon $F$ for valuable comments and suggestions provided.

Organon F 26 (4) 2019: 542-571 


\section{References}

Barrett, Jeffrey A. 2006. "A Quantum-Mechanical Argument for Mind-Body Dualism." Erkenntnis 65 (1): 97-115. https://doi.org/10.1007/s10670-006-9016-z

Bishop, Robert C. 2006. "The Hidden Premiss in the Causal Argument for Physicalism." Analysis 66 (289): 44-45. https://doi.org/10.1111/j.14678284.2006.00588.x

Bokulich, Peter. 2011. "Hempel's Dilemma and Domains of Physics." Analysis 71 (4): 646-51. https://doi.org/10.1093/analys/anr087

Braddon-Mitchell, David, and Frank Jackson. 1996. The Philosophy of Mind and Cognition. Cambridge, MA: Blackwell Publishers.

Bronowski, Jacob. 1970. "New Concepts in the Evolution of Complexity." Synthese 21 (2): 228-46. https://doi.org/10.1007/BF00413548

Chalmers, David J. 2006. "Strong and Weak Emergence." In The Re-Emergence of Emergence, edited by Philip Clayton and Paul Davies, 244-56. Oxford University Press. https://doi.org/10.1093/acprof:oso/9780199544318.003.0011

Crane, Tim, and D. H. Mellor. 1990. "There Is No Question of Physicalism." Mind 99 (394): 185-206. https://doi.org/10.1093/mind/XCIX.394.185

Crook, Seth, and Cart Gillett. 2001. "Why Physics Alone Cannot Define the 'Physical': Materialism, Metaphysics, and the Formulation of Physicalism." Canadian Journal of Philosophy 31 (3): 333-60. https://doi.org/10.2307/40232121

Dowell, Janice L. 2006. "The Physical: Empirical, not Metaphysical." Philosophical Studies 131 (1): 25-60. https://doi.org/10.1007/s11098-005-5983-1

Elsasser, Walter M. 1958. The Physical Foundation of Biology: An Analytical Study. London, NY, Paris and Los Angeles: Pergamon Press.

Elsasser, Walter M. 1962. "Physical Aspects of Non-Mechanistic Biological Theory." Journal of Theoretical Biology 3 (2): 164-91. https://doi.org/10.1016/S0022-5193(62)80013-7

Fiorese, Raphaël. 2015. "Stoljar's Dilemma and Three Conceptions of the Physical: A Defence of the Via Negativa." Erkenntnis 81 (2): 201-29. https://doi.org/10.1007/s10670-015-9735-0

Gatherer, Derek. 2008. "Finite Universe of Discourse. The Systems Biology of Walter Elsasser (1904-1991)." Open Biology Journal 1: 9-20. https://doi.org/10.2174/1874196700801010009

Gillett, Carl, and D. Gene Witmer. 2001. "A 'Physical Need': Physicalism and the Via Negativa." Analysis 61 (4), 302-09. https://doi.org/10.1093/analys/61.4.302

Hemmo Meir, and Orly R. Shenker. 2012. The Road to Maxwell's Demon. Cambridge: Cambridge University Press.

Hempel, Carl Gustav. 1966. Philosophy of Natural Science. Englewood Cliffs, NJ: Prentice-Hall. 
Kim, Jaegwon. 2006. "Emergence: Core Ideas and Issues." Synthese 151 (3): 54759. https://doi.org/10.1007/s11229-006-9025-0

Kripke, Saul. 1980. Naming and Necessity. Cambridge, MA: Harvard University Press.

Lewis, David. 1983/1999. "New Work for a Theory of Universal." Reprinred in Papers in Mataphysics and Epistemology, 8-55. Cambridge: Cambridge University Press.

Maudlin, Tim. 1996. "On the Unification of Physics." The Journal of Philosophy 93 (3): 129-44.

Meehl, Pauk E., and Wilfred S. Sellars. 1956. "The Concept of Emergence." In Minnesota Studies in the Philosophy of Science, vol. 1, edited by Herbert Feigl and Michael Scriven, 239-52. Minneapolis: University of Minnesota Press.

Montero, Barbara. 2001. "Post-Physicalism." Journal of Consciousness Studies 8 (2): 61-80.

Montero, Barbara, and David Papineau. 2005. "A Defense of the Via Negativa Argument for Physicalism." Analysis 65 (287): 233-37. https://doi.org/10.1093/analys/65.3.233

Ney, Alyssa. 2008. "Physicalism as an Attitude." Philosophical Studies 138 (1): 115. https://doi.org/10.1007/s11098-006-0006-4

Papineau, David. 2001. "The Rise of Physicalism." In Physicalism and Its Discontents, edited by Carl Gillett and Barry Loewer, 3-36. Cambridge: Cambridge University Press. https://doi.org/10.1017/CBO9780511570797.002

Papineau, David. 2016. "Naturalism." The Stanford Encyclopedia of Philosophy (Winter 2016 Edition), edited by Edward N. Zalta. URL = $<$ https://plato.stanford.edu/archives/win2016/entries/naturalism/>.

Prelević, Duško. 2017. "Hempel's Dilemma and Research Programmes: Why Adding Stances Is Not a Boon." Organon F 24 (4): 487-510.

Prelević, Duško. 2018. "Physicalism as a Research Programme." Grazer Philosophische Studien 95 (1): 15-33. https://doi.org/10.1163/18756735-000023

Redhead, Michael. 1996. From Physics to Metaphysics. Cambridge: Cambridge University Press. https://doi.org/10.1017/CBO9780511622847

Rescher, Nicholas. 1999. The Limits of Science. Pittsburgh: University of Pittsburgh Press. https://doi.org/10.2307/2181922

Robinson, William. 2015. "Epiphenomenalism." The Stanford Encyclopedia of Philosophy (Fall 2015 Edition), edited by Edward N. Zalta. URL = $<$ https://plato.stanford.edu/archives/fall2015/entries/epiphenomenalism/>.

Spurrett, David. 2017. "Physicalism as an Empirical Hypothesis." Synthese 194 (9): 3347-60. https://doi.org/10.1007/s11229-015-0986-8 
Spurrett, David, and David Papineau. 1999. "A Note on the Completeness of 'Physics'." Analysis 59 (261): 25-29. https://doi.org/10.1111/1467-8284.00144

Stoljar, Daniel. 2010. Physicalism. London and New York: Routledge.

Stoljar, Daniel. 2017. "Physicalism." The Stanford Encyclopedia of Philosophy (Winter 2017 Edition), edited by Edward N. Zalta. URL = $<$ https://plato.stanford.edu/archives/win2017/entries/physicalism/>.

Teller, Paul. 1992. "A Contemporary Look at Emergence." In Emergence or Reduction?, edited by Ansgar Beckermann, Hans Flohr and Jaegwon Kim, 13953. Berlin: De Gruyter.

Tononi, Giulio. 2008. "Consciousness as Integrated Information: A Provisional Manifesto." The Biological Bulletin 215 (3): 216-42. https://doi.org/10.2307/25470707

Vicente, Agustín. 2011. "Current Physics and 'the Physical'." The British Journal for the Philosophy of Science 62 (2): 393-416. https://doi.org/10.1093/bjps/axq033

Wilson, Jessica. 2006. "On Characterizing the Physical." Philosophical Studies 131 (1): 61-99. https://doi.org/10.1007/s11098-006-5984-8

Worley, Sara. 2006. "Physicalism and the Via Negativa." Philosophical Studies 131 (1): 101-26. https://doi.org/10.1007/s11098-005-5985-z 